\title{
La enseñanza virtual a través de videoconferencias como alternativas a la enseñanza de la medicina de pregrado y educación continua en época del COVID-19
}

\section{Virtual teaching through videoconferences as alternatives to the teaching of undergraduate medicine and continuing education in the time of COVID-19}

\author{
Juan Carlos Vázquez-Minero, ${ }^{*, \neq}$ María de Jesús Villalba-Calderón, ${ }^{\ddagger}$ Arturo Chávez-Tinoco,* \\ Carlos Daniel Vera-Márquez, Derly Emmanuel Fuentes-Gómez
}

\footnotetext{
*Instituto Nacional de Enfermedades Respiratorias Ismael Cosío Villegas (INER), México; ${ }^{\ddagger}$ Universidad Nacional Autónoma de México (UNAM), México; ${ }^{\circledR}$ Secretaría de Salud, México; "Cruz Roja Mexicana, México.
}

Palabras clave: Educación de pregrado, educación médica continua, videoconferencia, COVID-19.

Keywords: Undergraduate education, continuing medical education, video conferencing, COVID-19.

\section{INTRODUCCIÓN}

Desde sus inicios, la educación médica ha sufrido muchos cambios, tanto en los conceptos como en los escenarios educativos. Del hospital al aula, de la educación al pie del paciente $y$, finalmente, a los simuladores. Todos ellos responden a las necesidades de aprendizaje de los alumnos, de las necesidades educativas de la época y de las instituciones formadoras, los hospitales y las universidades.

Los avances del conocimiento del hombre también han influido en la educación y, por supuesto, en la enseñanza médica. En el siglo XX, el advenimiento de las computadoras con sus programas cada vez más amigables fueron adoptados por la medicina para crear cursos de educación médica, cuya base era la tecnología. Pero todo esto estaba

Correspondencia:

Dr. Juan Carlos Vázquez-Minero

Instituto Nacional de Enfermedades Respiratorias

Ismael Cosío Villegas, Ciudad de México, México.

Correo electrónico: minerojc@hotmail.com

Recibido: 07-IV-2021; aceptado: 07-V-2021.

Citar como: Vázquez-Minero JC, Villalba-Calderón MJ, Chávez-Tinoco A, Vera-Márquez CD, Fuentes-Gómez DE. La enseñanza virtual a través de videoconferencias como alternativas a la enseñanza de la medicina de pregrado y educación continua en época del COVID-19. Neumol Cir Torax. 2021; 80 (2): 84-88. https://dx.doi.org/10.35366/100987 diseñado como complemento de la educación «formal» de la medicina. Siempre la piedra angular y el centro de la enseñanza fue la relación personal directa del profesor, ya sea de ciencias básicas o de clínica con los alumnos. Una enseñanza eminentemente tutorial.

Y llegó el siglo XXI con más tecnología, con más recursos para la educación, con plataformas de comunicación más avanzadas que servían de manera parcial al contacto con la medicina. Todos los cursos y congresos eran presenciales, dándole mucho peso a la asistencia física del alumno.

Hasta ese momento pensar en comunicarse por vía virtual de manera cotidiana no era la primera opción. Iniciaba en la medicina la denominada «telemedicina» en algunos hospitales con la finalidad de dar consulta a distancia a través de un programa de videollamada.

Estas herramientas se empezaron a emplear de manera emergente debido a la crisis educativa que causó la pandemia de COVID-19, como una forma de cumplir el programa académico en el internado médico y en los cursos de educación continua.

A continuación, presentamos la experiencia de tres cursos dependientes de la Universidad Nacional Autónoma de México (UNAM) de la Ciudad de México; dos de pregrado en medicina y enfermería, y uno de educación médica continua para la preparación del examen para la residencia médica.

Las videoconferencias en el internado de pregrado en un instituto de salud durante el COVID-19

El internado médico de pregrado de la UNAM derivado del programa académico 2010 de la Facultad de Medici- 
na, contempla rotaciones clínicas en las áreas de cirugía, medicina interna, pediatría, ginecobstetricia, urgencias y medicina general. El Instituto Nacional de Enfermedades Respiratorias Ismael Cosío Villegas (INER), a raíz de la pandemia, se reconvirtió en un centro de atención exclusiva de pacientes con COVID-19; por lo que tuvo, por seguridad de los alumnos, que mover sus rotaciones clínicas a centros de salud y hospitales no COVID-19.

Así, para ciertas rotaciones, y en especial las de medicina interna, urgencias, pediatría y cirugía fue necesario utilizar las plataformas de videoconferencias para cumplir el programa académico en su parte teórica, con la finalidad de tener sesiones de casos clínicos en tiempo real y sincrónico. El uso de la plataforma fue por el tiempo de las dos rotaciones de internos en la materia de cirugía, con un total de cuatro meses, con dos horas a la semana, para 12 alumnos totales, complementadas por tareas enviadas y revisadas en plataformas de educación. Los resultados de las rotaciones fueron adecuados cumpliéndose el programa académico de la materia.

Se utilizó la plataforma Zoom y en todos los casos se usaron computadoras personales. Se hizo uso de las herramientas del chat y preguntas y respuestas para la interacción de los alumnos con sesiones no mayores a una hora. Por las condiciones de la pandemia no fue posible la realización de fases prácticas, sólo revisión de casos clínicos. La manera de evaluar el proceso educativo fue a través del examen departamental, en el cual el promedio fue de 8.5, un poco inferior a los resultados previos, que fueron de 9.0. En este grupo, no hubo problemas de conectividad técnicos, ni quejas por parte de los alumnos. En relación al proceso teórico, éste puede ser equivalente a la enseñanza presencial.

Las videoconferencias en el pregrado de la licenciatura de enfermería en un instituto nacional de salud durante el COVID-19

La licenciatura de enfermería de la Escuela de Enfermería Aurelia Saldierna Rodríguez del INER tuvo también que transformar sus escenarios, en el caso de las materias básicas y en particular la de procesos biofisiológicos I. La materia de manera tradicional era presencial, con un total de 8 horas a la semana. Se utilizaron las plataformas de videoconferencias con reuniones de 3 horas a la semana, para un total de 23 alumnos durante un semestre. Los trabajos se complementaron con interacción mediante plataformas de aulas virtuales. Tuvieron un adecuado desempeño los alumnos con participación activa en las clases, preguntas y repuestas. Se apoyó mediante la plataforma Cisco-WebEx. Se usaron computadoras personales y el teléfono celular en algunos alumnos con problemas de conexión. Se utilizaron las herramientas del chat para la comunicación y sesión de preguntas y respuestas. Los tiempos de cada sesión fueron de una hora.

En este grupo no pudieron realizarse las prácticas de laboratorio programadas, se sustituyeron con la utilización de videos o de revisión en teoría de los temas. La evaluación se llevó a cabo mediante exámenes parciales con formatos de Google Forms, con resultados en promedio de 8 (semejantes a los grupos con clases normales). Sólo un alumno se fue a examen final. En este grupo, algunos alumnos usaron teléfono celular para conectarse, hubo dos con problemas técnicos para la conexión, pero no fue cotidiano.

\section{Las videoconferencias en el curso de preparación para el Examen Nacional para Aspirantes a Residencias Médicas (ENARM) durante el COVID-19}

Para comparar con el uso de las videoconferencias en materias de pregrado se tomó el ejemplo de un curso preparatorio para el examen de residencias. El ENARM es una evaluación de aplicación anual dirigida y regulada por la Comisión Interinstitucional para la Formación de Recursos Humanos para la Salud (CIFRHS), cuyo objetivo es establecer una selección de candidatos con el mayor puntaje para obtener un lugar dentro de una especialidad médica elegida por cada uno.

A lo largo del tiempo se han creado cursos de preparación que están enfocados a adquirir y fortalecer los conocimientos que permitan resolver los casos clínicos en los aspectos de diagnóstico y tratamiento que se presentan en el ENARM. Uno de estos cursos lo imparte la Facultad de Medicina de la UNAM, de manera tradicional éstos eran presenciales a lo largo de períodos previos. Este curso tipo diplomado para la sistematización de conocimientos médicos para la preparación del examen de residencias médicas tiene una duración de 120 horas y tradicionalmente es presencial. Debido a la pandemia fue necesario reconvertirlos y hacerlos a distancia para guardar las medidas de prevención de transmisión del COVID-19.

Por lo tanto, fue necesario utilizar plataformas de videoconferencias en tiempo real para poder cumplir con el programa académico de éstos. Los profesores tuvieron que transformar sus presentaciones presenciales en reuniones, cuyo formato era dado entre el grupo de alumnos, el profesor y como vía de comunicación la plataforma digital de videoconferencia en tiempo real y sincrónico. En el caso particular del módulo de otorrinolaringología fueron dos cursos, con un total de 6 horas cada uno con una asistencia de 67 y 65 en cada curso, con buena respuesta y participación de los alumnos, complementando las pláticas con sesiones de preguntas y respuestas, aprovechando también el recurso del chat que tiene esta plataforma de videoconferencia. Esto fue llevado a cabo mediante la plataforma Zoom que utiliza la UNAM. En la mayoría de los casos se 
usaron computadoras personales y en algunos tabletas y celulares. Este curso al ser sólo una parte del mismo y por su diseño de un solo día no tuvo evaluación. No existieron problemas de conexión por parte de los alumnos. En relación al proceso teórico, éste puede ser equivalente que al de la enseñanza presencial.

\section{Aplicación de la enseñanza virtual a través de videoconferencias}

Pensar en comunicarse a distancia por audio y video en tiempo real hace algunos años era cuestión reservada para la televisión. En el ámbito educativo no existía una manera de hacerlo a distancia, había métodos como la telesecundaria, que en realidad eran videos pregrabados para completar la educación en ciertas escuelas de nivel medio.

Con los avances de la tecnología se diseñaron softwares que acoplaban audio y video para hacer conexiones en tiempo real y a éstas se les denominó videoconferencias. Por tanto, en la actualidad, la videoconferencia es el conjunto de hardware y software que permiten la conexión simultánea por medio de audio y video, para el intercambio de información interactiva en tiempo real entre personas que se encuentran geográficamente distantes. ${ }^{1}$

Estas videoconferencias se pueden dar desde una computadora personal, tableta, o bien, desde un teléfono celular teniendo la aplicación correspondiente, sin tener ningún problema salvo la conectividad y capacidades de las redes. ${ }^{2}$

Las limitantes que se pueden tener en la utilización como herramienta de educación es la accesibilidad a los equipos de cómputo por parte de los alumnos. Se sabe que en personas de ingreso bajo sólo 1.2\% tiene una computadora personal y entre personas de ingreso alto el porcentaje es de $60 \%{ }^{3}$

Los cursos en línea apoyados en videoconferencias son formatos para aprender virtualmente de manera sincrónica. El punto básico de este modelo que no es el único, es el profesor como centro de la enseñanza, siendo un experto e instructor y que ha planeado de manera correcta y útil el curso. Estos cursos son estructurados en tres modelos de presentación, interacción y colaboración. En el primero es una presentación por parte del profesor de manera unidireccional. El segundo cuenta con una interacción entre el alumno y el curso con preguntas y respuestas, ejercicios en línea y el tercero con intercambio de información por parte de los alumnos. ${ }^{3}$

La realidad es que cada vez más médicos y profesores utilizan el Internet para la búsqueda e intercambio de información, se cree que hasta 99\% cuenta con estas herramientas; y esto impacta mucho en esta etapa en la educación y los cuidados de la salud. El uso de sitios web y redes del Internet y plataformas multimedia con fines educativos en la medicina es de hasta 59\%.,
Un requisito indispensable es que en estos cursos el profesor o tutor sea un buen presentador, con ello se garantiza una parte del curso que es la adecuada comunicación de las ideas. ${ }^{6}$

\section{Habilidades docentes con el uso de plataformas de videoconferencias}

Cuando llegó la pandemia de COVID-19 provocó que las escuelas y facultades de medicina interrumpieran sus actividades y reconvirtieran sus métodos educativos a las medidas de distanciamiento social. Tuvieron que mantener los procesos formativos de la licenciatura, de los posgrados y de la educación continua. Para ello tuvieron la necesidad de utilizar recursos tecnológicos que antes sólo se usaban de manera esporádica y como complemento de la educación. Las clases presenciales se transformaron en videoconferencias. Es importante destacar que la mayoría de la plantilla docente está formada por profesores que no son nativos digitales y se muestran reacios a utilizar las tecnologías de la información y la comunicación (TIC). ${ }^{7}$

Esta migración hacia la virtualidad se ha denominado «educación remota de emergencia», en donde los grupos pueden tomar sus clases desde su casa, sin arriesgar el contagio por contacto social. Esta modalidad involucra el uso de la enseñanza totalmente remota, a través de videoconferencias para las sesiones sincrónicas y apoyo con otras plataformas para actividades y tareas. ${ }^{8}$

Para poder llevar a cabo esta educación emergente es necesario que el profesor se familiarice con las múltiples plataformas que pueden ayudar a la educación, son diversas, pero en particular utilizamos Zoom y Cisco-WebEx de acuerdo con las especificaciones de cada institución. Es importante, además, que las sesiones sean de entre 45 minutos a una hora, con un tiempo para preguntas y respuestas. También que durante las sesiones exista retroalimentación de los conceptos y tener muy claro las estrategias de evaluación. ${ }^{9}$

Tal vez una de las preocupaciones más importantes con el uso de las videoconferencias es ser un «espejo del aula presencial», es decir, donde el docente sea meramente un expositor sin interacción con el alumno, que se pierda la posibilidad de un adecuado intercambio de ideas y reforzamiento del aprendizaje. En los tres escenarios que aquí presentamos se pudo realizar el intercambio de ideas a través de sesión de preguntas, o bien mediante el chat. 10,11

La videoconferencia debe brindar al docente una gran experiencia para motivarlo a conectarse a distancia con los alumnos y poder tener una interacción en tiempo real y de manera sincrónica con el fin de cumplir la labor docente, si bien es cierto que esta interacción dependerá también de las habilidades que vaya desarrollando a lo largo del uso de estas plataformas. En nuestras tres experiencias la 
interacción fue buena y con resultados muy productivos para los alumnos. ${ }^{11}$

\section{Recomendaciones del uso de videoconferencias}

El papel que juega el docente en la actualidad es el de ser un gran activista del aprendizaje, no ser el centro de la enseñanza, sino impulsar al alumno a conseguir sus metas educativas. Al presentarse este cambio en la manera de enseñar y utilizar la tecnología como una herramienta importante en este proceso, hay que destacar que el profesor debe ser un incitador del proceso de aprendizaje durante la videoconferencia, impulsando al alumno a interactuar con él y entre los mismos alumnos para poder completar los procesos de comunicación y aprendizaje. No deberá ser solamente un expositor y una persona que resuelva dudas. Podremos utilizar los chats, foros, correos, llamadas y mensajería instantánea para introducir a la videoconferencia preguntas detonadoras, enlaces que completen el tema, en fin, cualquier recurso capaz de despertar el interés del alumno.

Al utilizar herramientas como la videoconferencia, es indispensable que el docente conozca los recursos como son el software, la cámara digital y el micrófono de los diferentes dispositivos, y tratar de optimizar éstos en cada uno de los dispositivos, como es la computadora personal y el teléfono celular. Otros elementos básicos a considerar son la red de comunicación y la sala donde se va a trasmitir, la cual debe contar con una adecuada iluminación hacia el docente, a fin de que su imagen se trasmita sin sombras y de manera clara. ${ }^{12}$

Un punto importante también es la manera de trasmitir los contenidos que deben ser claros, directos y con adecuada retroalimentación en la clase y con trabajos en casa que permitan reforzar lo hecho durante la videoconferencia. La limitante importante de esto en la medicina son las prácticas clínicas, las cuales son difíciles de sustituir con educación a distancia. ${ }^{13}$

Lo que esta pandemia ha enseñado al profesor es convertir su aula presencial en una virtual, hacer que la comunidad educativa presencial se convierta en un grupo de personas unidas por un sistema digital y que a pesar de la distancia sea una adecuada interacción en un ambiente de aprendizaje. Reconocer que la tecnología no desplaza al docente sino que lo apoya, y que el docente debe jugar el doble papel de ser el centro de la enseñanza en momentos, a ser el incitador de la educación en donde el centro es el alumno. ${ }^{14}$

Finalmente, es necesario destacar que esta pandemia en lugar de detener el proceso de educación lo transformó de manera positiva, utilizando herramientas que antes no se ocupaban en su totalidad y haciendo que nuestros sistemas educativos se refuercen y fortalezcan. Las instituciones edu- cativas de pregrado y educación continua pueden reunir de manera sincrónica a un grupo variado de personas que están a muchos kilómetros de distancia, con el fin de llevar a cabo un adecuado proceso de enseñanza-aprendizaje. ${ }^{15,16}$

\section{CONCLUSIONES}

La enseñanza virtual a través de videoconferencias en época del COVID-19 ha sustituido de manera eficaz a las clases presenciales, pudiendo haber una interacción con sana distancia de los participantes. Ha requerido una adaptación a la mentalidad y habilidades por parte de los profesores y a un cambio de actitud por parte de los alumnos.

\section{REFERENCIAS}

1. Acosta RMR, Castro ME, Frías LMG, Reyes MMR, Acosta AG. Uso de las tecnologías en telecomunicación para la videoconferencia dual con aplicación en la medicina. Evid Med Invest Salud. 2015;8(1):28-31.

2. Case T, Morrison C, Vuylsteke A. The clinical application of mobile technology to disaster medicine. Prehosp Disaster Med. 2012;27(5):473-480. Available in: https://doi.org/10.1017/ s1049023x12001173

3. Mendoza LE. Aprendizaje electrónico: su papel en la educación médica continua. Ped Mex. 2011;13(3):127-133.

4. Steinbrook R. Searching for the right search--reaching the medical literature. N Engl J Med. 2006;354(1):4-7. Available in: https://doi. org/10.1056/nejmp058128

5. Landgrave IS, Ponce RE, Baillet EL, Irigoyen CA, Jiménez GI, Sámano $S A$. Uso de la web e internet como herramienta para la búsqueda de información médica científica. Arch Med Fam. 2016;18(4):95-106.

6. Bidarra J, Mason R. El potencial del video en la educación abierta y a distancia. Rev Iberoame Educ Dist. 1998;1(2):101-115.

7. Abreu-Hernández LF, León-Bórquez R, García-Gutiérrez JF. Pandemia de COVID-19 y educación médica en Latinoamérica. FEM. 2020;23(5):237-242.

8. Hodges C, Moore S, Lockee B, Trust T, Bond A. The difference between emergency remote teaching and online learning. Access date: 2020 August 23. Available from: https://er.educause.edu/ articles/2020/3/the-difference-between-emergency-remote-teachingand-online-learning

9. Vásquez D. Ventajas, desventajas y ocho recomendaciones para la educación médica virtual en tiempos de COVID-19: Revisión de tema. Rev CES Med. 2020;34:14-27. Especial COVID-19.

10. De Vincenzi A. Del aula presencial al aula virtual universitaria en contexto de pandemia de COVID-19. Debate Universitario [Internet]. 2020 [Fecha de acceso 12 de agosto de 2020] Disponible en: https:// uai.edu.ar/media/115153/4-del-aula-presencial-al-aula-virtualuniversitaria-en-contexto-de-pandemia-covid-19.pdf

11. Chambi ME. La videoconferencia como recurso educativo en los tiempos del COVID-19. Inv Ed Med. 2020;9(36):108-109.

12. Chacón MA. La videoconferencia: conceptualización, elementos y uso educativo. Rev Etica@net [Internet]. 2003;1(2):1-13. Disponible en: www.ugr.es/local/sevimeco/revistaeticanet/index.htm

13. Palés-Argullós J, Gomar-Sancho C. Repercusiones de la COVID-19 en la educación de los médicos: la formación de grado (II). FEM (Ed. impresa) [Internet]. 2020;23(4): 161-166. Disponible en: 
http://scielo.isciii.es/scielo.php?script=sci_arttext\&pid=S201498322020000400001\&lng=es

14. Mendoza-Castillo L. Lo que la pandemia nos enseñó sobre la educación a distancia. RLEE [Internet]. 2020;50(Especial):343-352. Disponible en: https://rlee.ibero.mx/index.php/rlee/article/view/119

15. Núñez-Cortés JM. Educación médica durante la crisis por COVID-19. Educ Med. 2020;21(3):157. Disponible en: http://dx.doi.org/10.1016/j. edumed.2020.05.001
16. Núñez-Cortés JM, Reussi R, Garcia DM, Falasco S. COVID-19 y la educación médica, una mirada hacia el futuro. Foro lberoamericano de Educación Médica (FIAEM). Educ Med. 2020;21(4):251-258. Disponible en: http://dx.doi.org/10.1016/j.edumed.2020.06.004

Conflicto de intereses: Los autores declaran no tener conflicto de intereses. 